\title{
Construction of Recombinant Bacmid Containing M2e-Ctxb and Producing the Fusion Protein in Insect Cell Lines
}

\author{
Nima Mirzaei ${ }^{1, ~}$; Talat Mokhtari Azad ${ }^{2}$; Rakhshandeh Nategh ${ }^{2}$; Hoorieh Soleimanjahi ${ }^{3}$; \\ Nour Amirmozafari ${ }^{4}$ \\ ${ }^{1}$ Department of Biology, Science and Research branch, Islamic Azad University, Tehran, IR Iran \\ ${ }^{2}$ Department of Virology, Tehran University of Medical Sciences, Tehran, IR Iran \\ ${ }^{3}$ Department of Virology, Tarbiat Modares University, Tehran, IR Iran \\ ${ }^{4}$ Department of Microbiology, Tehran University of Medical Sciences, Tehran, IR Iran \\ *Corresponding Author: Nima Mirzaei, Department of Biology, Science and Research branch, Islamic Azad University, Tehran, IR Iran. Tel: +98-2188962343, E-mail: nm_mirzaei@ \\ yahoo.com \\ Received: June 30, 2013; Revised: July 27, 2013; Accepted: November 18, 2013
}

\begin{abstract}
Background: Sequence variations in glycoproteins of influenza virus surface impel us to design new candidate vaccines yearly. Ectodomain of influenza M2 protein is a surface and highly conserved protein. M2e in influenza vaccines may eliminate the need for changing vaccine formulation every year.

Objectives: In this study, a recombinant baculovirus containing M2e and cholera toxin subunit B fusion gene was generated with transposition process to express in large amounts in insect cell lines.

Materials and Methods: M2e-ctxB fusion gene was created and cloned into pFastBac HT. The recombinant vector was transformed into DH1OBac cells to introduce the fusion gene into the bacmid DNA via a site-specific transposition process. The recombinant bacmid was then extracted from white colonies and further analyzed using PCR, DNA sequence analyzing, and indirect immunofluorescence assay. Results: PCR and DNA sequence analyzing results showed that the fusion gene was constructed as a single open reading frame and was successfully inserted into bacmid DNA. Moreover, indirect immunofluorescence results showed that the fusion gene was successfully expressed.

Conclusions: Baculovirus expression vector system is valuable to produce M2e based influenza vaccines due to its simple utilization and ease of target gene manipulation. The expressed protein in such systems can improve the evaluating process of newvaccination strategies. Keywords:Influenza Vaccines; Baculovirus; Cholera Toxin Subunit B
\end{abstract}

\section{Background}

Influenza is one of the most serious public health predicaments in the world (1-4) Influenza virus A has a potential ability to cause epidemic and pandemic flu via two relatively close manners. Surface antigenic variations, commonly named as antigenic drift, and reassortment of RNA segments among different circulating strains of the virus commonly named as antigenic shift $(5,6)$ are the two mechanisms. These variations usually occur yearly due to the circulation of the virus among the population allowing the virus to escape host acquired and innate immune responses. Such abilities coerce us to design and change influenza vaccine formulations every year $(2,7)$. Many investigations have been performed to design and produce more effective vaccines to prevent spreading pandemic influenza (8-10). An effective influenza vaccine should be developed based on the genetically stable antigens (11). One of the most conserved influenza antigens is external-domain of M2, briefly named as M2e. M2 helps to translocate $\mathrm{H}+$ ions to endocytosed virions and acts as a modulator of $\mathrm{pH}$ (12-14). Despite the genetic stability, M2e weak immunogenicity is the most important concern for using it as an influenza vaccine. It usually fails to illicit an effective immune response against the virus (6).

Using adjuvants and genetic tags to other potent immunogens such as heat shock protein 70 is suggested to enhance M2e immunogenicity (15). Cholera toxin subunit B (CtxB) would be an applicable antigen to develop fusion vaccines due to its potential ability to induce mucosal immune responses (16). For evaluating efficiency and immunogenicity of fusion vaccines, a large amount of recombinant antigen should be provided. Different types of expression systems have been developed to produce recombinant proteins. Prokaryotic and eukaryotic expression systems are the two major well-characterized expression systems widely used to produce recombinant proteins. Prokaryotic expression systems apply bacterial would not be effective in designing influenza virus vaccine due to its low level immunogenicity. However, applying M2e along with the potent immunogens such as ctxB would increase the efficacy of M2e based influenza vaccines. The expression system is important to achieve reliable results in vaccine research. Baculovirus expression system is appropriate to produce fusion proteins due to its ability to produce recombinant protein in conditions similar to the human cells and many other advantages.

Copyright (C) 2014, Iranian Red Crescent Medical Journal; Published by Kowsar Corp. This is an open-access article distributed under the terms of the Creative Commons Attribution License, which permits unrestricted use, distribution, and reproduction in any medium, provided the original work is properly cited. 
cells as host and mainly used for production of rather uncomplex protein and peptides. Eukaryotic expression systems have been studied in the last decades, mainly based on yeast, mammalian or insect cells to produce complex recombinant proteins. Although prokaryotic systems can be useful for protein production, these systems have some limitations. For example, they cannot glycosylate the expressed proteins and therefore some of eukaryotic proteins expressed in E. coli and other prokaryotic systems are non-functional. Moreover, the bacterial expressed proteins are usually mixed with endotoxin derived from the bacterial cell wall. These limitations have many implications in downstream process and studies such as vaccine research (17). It has become a common expression system for both basic research and large-scale commercial applications. A key factor to the popularity of insect cell expression is the ability of insect cells to produce relatively large quantities of post-translationally modified eukaryotic proteins in a relatively short period. Most insect cell-produced proteins have been expressed by employing the Baculovirus Expression Vector System (BEVS). BEVS is suitable to produce large amounts of recombinant protein in insect cell lines such as Sf9 and Sf21. The BEVS is an approved system for heterologous expression and production of viral antigens with vaccine potential for humans and animals. BEVS has been widely used for production of subunit vaccines against parasitic diseases as well. BEVS uses transport system present in higher eukaryotic cells providing conditions more similar to the human cells. Moreover, there are a wide variety of vectors and culture media adapted for this system making it simple to use $(18,19)$.

Different vectors have been developed for expressing the target gene in insect cell lines. Most BEVSs use Autographa californica Multiple Nuclear Polyhedrosis Virus (AcMNPV) as the prototype baculovirus. Due to the large size of the AcMNPV genome ( $134 \mathrm{~Kb}$ ), a homologous recombination or transposition is required to insert the target gene into the baculovirus genome. In practice, the target gene is subcloned into a transfer vector containing a suitable promoter flanked by a part of baculovirus DNA derived from non-essential locus such as the polyhedrin gene of AcMNPV to perform recombination with the baculovirus genome. The recombinant viral DNA is then transfected into the insect cell lines. To do this process easily, the baculovirus genome has been modified, so that it can be maintained in E.coli. This form of baculovirus DNA which can be maintained and amplified in both E.coli and insect cell is named bacmid. Moreover, recently manipulated baculovirus genome (bacmid) has other features such as the ability to perform site-specific transposition for faster inserting the foreign gene, by adding Tn7, and ease of selection, by introducing lacZ cassette into the bacmid DNA. The bacmids are usually carried by special strains of E. coli such as DH10Bac (20). Appearing such modified BEVSs help us to evaluate new generation of vaccines more effectively in comparison with other expression systems.

\section{Objectives}

The aim of this study was to produce recombinant M2ectxB fusion protein using Baculovirus Expression Vector System via a site-specific transposition able to insert the fusion gene into bacmid DNA and evaluating expression of the fusion protein in insect cell line by indirect immunofluorescence assay.

\section{Materials and Methods}

\subsection{Bacterial Strain and Viruses}

Toxigenic Vibrio cholera strain 569B was employed to amplify ctxB. Influenza A/Puerto Rico/8/34 was used as standard strain to amplify M2e. E.coli strain Top10 (F- end A1 recA1) (Invitrogen, USA) was used for transformation and amplifying the recombinant vector pFastBac HT/ M2e-ctxB. E.coli Top10 is an appropriate strain to perform the cloning process. Construction of the recombinant baculovirus genome containing M2e-ctxB (recombinant bacmid) was performed in E.coli strain DH10Bac (F-endA1 lacZ $\triangle$ M15) (Invitrogen, USA).

\subsection{Plasmid and Cloning Vectors}

To generate recombinant bacmid pFastBac HT was used as the transfer vector. E. coli strain DH1OBac contained the baculovirus modified DNA (bacmid) with a mini-attTn7 target site and the helper plasmid. The helper plasmid harbored by DH10Bac strains, confers resistance to tetracycline and encodes enzymes needed for transposition of the gene of interest onto the bacmid. Therefore, adding appropriate concentration of tetracycline into the agar plates is very important to maintain helper plasmid in $E$. coli DH10Bac. The transfer vector carried mini-Tn7 element, polyhedron promoter and $\mathrm{N}$-terminal $6 \mathrm{X}$ histidine tag.

\subsection{PCR Amplification}

M2e was amplified using influenza virus $\mathrm{A} /$ Puerto Rico/8/34 genome as the template with the following primers:

\section{F: 5'-CGGGATCCACCATGTCCCTGCTGACCGAGG-3' \\ R: 5'-CCCAAGCTTAGCCATCGCTGCTGCCATT-3'}

The forward primer contained BamHI restriction site (in bold) and start codon (the underlined nucleotides). The Reverse primer contained HindIII restriction site, shown in bold type. Oligo software V. 7 was used to design primers. The RT-PCR and PCR reaction was performed using super script III one step RT-PCR kit (Invitrogen, USA) in a single tube containing $25 \mu \mathrm{L}$ reaction mix, 1 unit RT/ Taq DNA polymerase, Template ( $1 \mu \mathrm{g}), 1 \mu \mathrm{L}$ of F and R primers (10 pmol for each), and water nuclease-free to $50 \mu \mathrm{L}$ final volume. The PCR program included the following 
steps: cDNA synthesis at $48^{\circ} \mathrm{C}$ for $30 \mathrm{~min}$, denaturation at $94^{\circ} \mathrm{C}$ for $2 \mathrm{~min}$, followed by 40 cycles of $94^{\circ} \mathrm{C}$ for $15 \mathrm{sec}$ (denaturation), $58^{\circ} \mathrm{C}$ for $30 \mathrm{sec}$ (annealing), $68^{\circ} \mathrm{C}$ for $1 \mathrm{~min}$ (extention), and a final extension at $68^{\circ} \mathrm{C}$ for $5 \mathrm{~min}$. Genomic DNA from Vibrio cholera 569B was isolated using Gene Jet genomic DNA purification kit(Thermo scientific, USA). The PCR reaction was performed using the primers F: 5'-CCCAAGCTTATTAAATTAAAATTTGGTG-3' R: 5'-CGGAATTCTTAATTTGCCATACTAATTG-3'

HindIII and EcoRI restriction sites (in bold) were added into the F and R primers, respectively. Oligo software V. 7 was used to design primers. PCR reaction was performed in a tube containing $5 \mu \mathrm{L}$ of 10x PCR buffer, $1 \mu \mathrm{L}$ of dNTP mix (0.2 mM for each), $1.5 \mu \mathrm{L}$ of $\mathrm{MgCl} 2(1.5 \mathrm{mM}), 1 \mu \mathrm{L}$ of each primer (10 pmol for each), 1-2 $\mu \mathrm{L}$ of template DNA, 1 unit of platinum Taq DNA polymerase (Invitrogen, USA) and water nuclease-free up to $50 \mu \mathrm{L}$ final volume. The PCR program included the following steps: denaturation at $94^{\circ} \mathrm{C}$ for $1 \mathrm{~min}$, followed by 30 cycles of $94^{\circ} \mathrm{C}$ for $30 \mathrm{sec}$ (denaturation), $62^{\circ} \mathrm{C}$ for $30 \mathrm{sec}$ (annealing), $72^{\circ} \mathrm{C}$ for $1 \mathrm{~min}$ (extension), and a final extension at $72^{\circ} \mathrm{C}$ for $5 \mathrm{~min}$. All the PCR products were then purified using PCR purification kits (Bioneer, South Korea) and sequenced.

\subsection{Fusion of M2e to $c t x B$}

The amplified M2e and ctxB were gel purified and digested with HindIII (Thermo scientific, USA) separately. The digested products were ligated using T4 DNA ligase (Invitrogen, USA) and incubated overnight at room temperature to create M2e-ctxB fusion gene. A PCR was performed directly on ligation product using M2e forward and ctxB reverse primers to amplify the fusion fragments. The amplified M2e-ctxB fragments were purified and sequenced using the BigDye $₫$ Terminator v3.1 Cycle Sequencing Kit (Invitrogen, USA) to confirm the accuracy of the fusion process.

\subsection{Cloning and Construction of pFastBac HT/ M2e-ctxB}

The PCR product was gel purified and double digested with BamHI and EcoRI (Thermo scientific, USA) and then ligated into BamHI and EcoRI pre-digested pFastBac HT (Invitrogen, USA). The ligation product was incubated overnight at room temperature and then used for transformation of E. coli strain Top10. The transformants were plated on LB agar plates containing $100 \mu \mathrm{g} / \mathrm{mL}$ ampicillin and incubated at $37^{\circ} \mathrm{C}$ for $24 \mathrm{~h}$.

\subsection{Generating Recombinant Bacmid Containing M2e-ctxB}

After cloning, E.coli strain DH10Bac cells which carried the bacmid DNA, as well as kanamycin and tetracycline resistance genes, were chemically transformed with 1ng of the purified pFastBac HT/M2e-ctxB. The recombinant bacmid was created by culturing the transformants in LB broth. Following incubation at $37{ }^{\circ} \mathrm{C}$ in a shaker $(225$ $\mathrm{rpm}$ ) for $4 \mathrm{~h}$ to allow expression of the antibiotic resistance genes, insertion of the mini-Tn7 containing M2ectxB from pFastBac HT into the mini-attTn7 attachment site on bacmid DNA was performed. After the incubation period serial dilutions $\left(10^{-1}, 10^{-2}, 10^{-3}\right)$ of the cells were prepared with LB broth and $100 \mu \mathrm{L}$ of each dilution was plated on LB agar containing $7 \mu \mathrm{g} / \mathrm{mL}$ gentamicin, $50 \mu \mathrm{g} /$ $\mathrm{mL}$ kanamycin, $10 \mu \mathrm{g} / \mathrm{mL}$ tetracycline, $40 \mu \mathrm{g} / \mathrm{mL}$ IPTG, and $100 \mu \mathrm{g} / \mathrm{mL}$ X-gal (Invitrogen, USA). The plates were incubated at $37^{\circ} \mathrm{C}$ for at least $48 \mathrm{~h}$ until the blue and white colonies were appeared. The white colonies were picked and restreaked on LB agar containing the above mentioned concentration of antibiotics, IPTG and X-gal.

\subsection{PCR Analyzing of the Recombinant Bacmid}

To verify successful insertion of the M2e-ctxB fusion gene into the bacmid DNA, the purified recombinant bacmid was analyzed by PCR. A single colony with a white phenotype was picked and inoculated into LB broth containing appropriate concentrations of kanamycin, gentamicin and tetracycline. Following incubation at $37{ }^{\circ} \mathrm{C}$ at $225 \mathrm{rpm}$ for 18-24 h, the cells were harvested and recombinant bacmid was purified manually according to the protocol provided by the manufacturer (Invitrogen, USA). Presence of the M2e-ctxB in bacmid DNA was verified by PCR using a combination of pUC/M13, gene specific primers and each set of primers lonely. pUC/M13 primers were custom synthesized with the following sequences:

pUC/M13 F: 5'-CCCAGTCACGACGTTGTAAAACG-3'

pUC/M13 R: 5'-AGCGGATAACAATTTCACACAGG-3'

PCR was performed according to the manufacturer's instructions and analyzed by agarose gel electrophoresis.

\subsection{Transfection and Infection of Sfg Cells by Re- combinant Bacmid}

The precultured Sf9 cells were diluted by serum free medium to $1 \times 10^{6}$ cells $/ \mathrm{mL}$ and then transfected by recombinant bacmid using Cellfectin II reagent (Invitrogen, USA). The recombinant bacmid was collected after $72 \mathrm{~h}$ according to the manufacturer instruction. To produce M2e-CtxB fusion protein, the Sfy cells were seeded at the density of $1-2 \times 10^{7}$ per flask $\left(75 \mathrm{~cm}^{2}\right)$ and infected by recombinant bacmid collected from the last stage at MOI (Multiplicity of Infection) 3 in $10 \mathrm{~mL}$ of Sf-900 III SFM (Invitrogen, USA). All the flasks were incubated at $27^{\circ} \mathrm{C}$.

\subsection{Indirect Immunofluorescence Assay}

To confirm expression of M2e-CtxB fusion protein, indirect immunofluorescence assay was performed using anti-beta subunit cholera toxin FITC conjugated antibody (Abcam, UK). At $6 \mathrm{~h}$ post-infection Sf 9 cells were washed with PBS and fixed with cold acetone. Production of M2e- 
CtxB fusion protein was detected by the anti-cholera toxin B subunit monoclonal antibody diluted in PBS. The antibodies were removed and cells were washed three times with PBS and then incubated with the secondary at $37^{\circ} \mathrm{C}$ for $20 \mathrm{~min}$. The cells were subsequently re-washed with PBS and visualized by immunofluorescence microspore.

\section{Results}

\subsection{PCR Amplifying of the M2e and $c t x B$}

The fragments M2e and ctxB were successfully amplified by PCR. The amplified M2e fragment was about 80 bp and ctxB amplified fragment was 380 bp. The amplified fragments were then fused to construct M2e-ctxB amplified by PCR. M2e-ctxB fusion fragment was about $460 \mathrm{bp}$. Sequence data showed that M2e was successfully attached to the 5 ' end of ctxB and a single open reading frame was generated.

\subsection{Cloning and Construction of pFastBac HT/ M2e-ctxB}

The amplified fusion fragments were then cloned into pfastBac HT to form pFastBac HT/M2e-ctxB. E.coli strains Top10 was transformed by pFastBac HT/M2e-ctxB and colony PCR was performed to confirm insertion of the M2e-ctxB fusion gene into pFastBac HT. All the selected colonies carried the recombinant pFastBac HT/M2e-ctxB based on the results of agarose gel electrophoresis. The amplified fragment was approximately $460 \mathrm{bp}$ in all the selected colonies (Figure 1). The sequence of fusion gene and accuracy of the cloning process were confirmed by DNA sequence analyzing. DNA Sequence analyzing showed that M2e was successfully placed in the 5 ' end of ctxB.

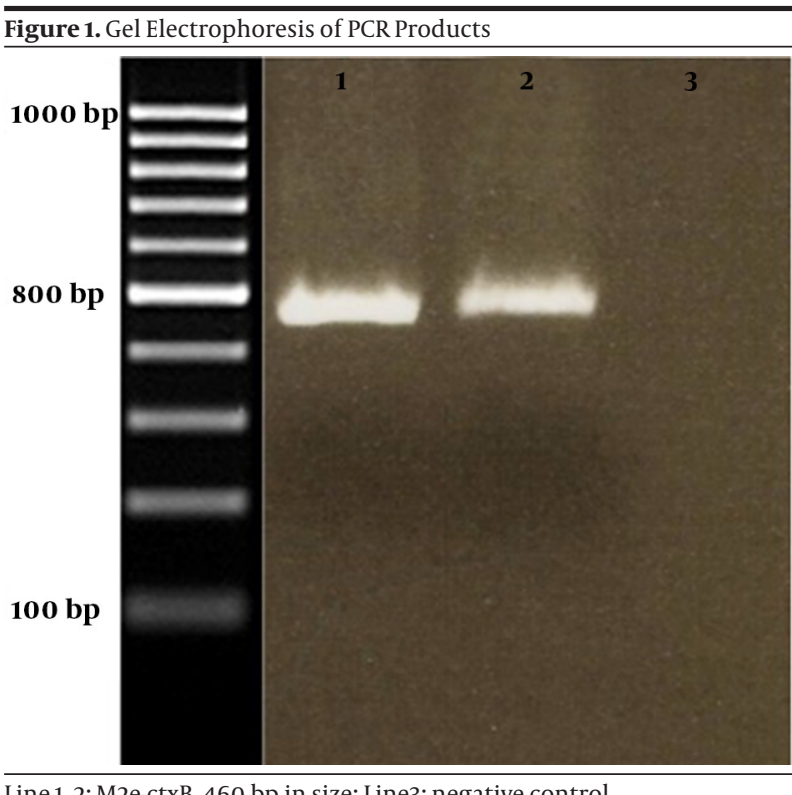

Line 1, 2: M2e-ctxB, 460 bp in size; Line3: negative control

\subsection{Construction of Recombinant Bacmid}

The purified recombinant pFastBac HT/M2e-ctxB was transformed into E.coli strain DH10Bac to generate recombinant bacmid containing M2e-ctxB. After $48 \mathrm{~h}$ incubation, the white and blue colonies appeared on plates containing kanamycin, tetracycline, gentamicin, X-gal and IPTG. Some white colonies were selected and restreaked on agar palates to ensure whether they show the white phenotype.

\subsection{PCR Analysis of Recombinant Bacmid}

We performed a series of PCR reactions for final confirmation of the recombinant bacmid construction. The PCR reactions were performed using a combination of backbone and gene specific primers to avoid any false results due to contamination. The amplified fragments using pUC/M13 F and M2e/ctxB R, M2e/ctxB F and pUC/M13 $\mathrm{R}$, pUC/M13, and M2e/ctxB forward and reverse primers were between 460 and 2900 bp in size. PCR reaction using non-recombinant bacmid DNA as template amplified pUC/M13 priming region. The corresponding bond on agarose gel was almost 300 bp in size (Figure 2). All primer sequences and the amplified fragments were shown in Table 1.

\subsection{Indirect Immunofluorescence Assay}

The infected Sf9 cells were analyzed by fluorescence microscopy to verify production of the recombinant protein. We successfully detected green fluorescence in Sfg cell infected with the recombinant bacmid. We also used the non-infected Sf9 cells as negative control to compare with the infected cells to evaluate green fluorescence emitting (Figure 3).

Figure 2. Pattern of PCR Reaction on Recombinant Bacmid Using a Combination of Different Primers

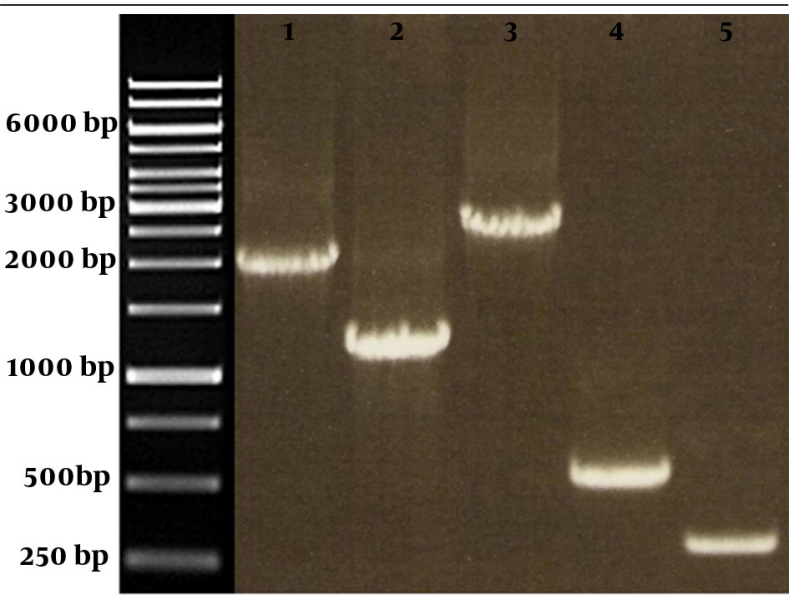

Lane1: PCR reaction using pUC/M13 F and M2e-ctxB R; Lane2: PCR reaction using M2e-ctxB F and pUC/M13 R; Lane 3: PCR reaction using pUC/M13 F and R; Lane4: PCR reaction using M2e-ctxB F and R; Lane5: PCR reaction using pUC/M13 F and R and non-recombinant bacmid as template. 
Mirzaei Net al.

\begin{tabular}{llll}
\hline \multicolumn{2}{l}{ Table 1. Details of Amplified Region and Primer Sets Used for PCR Analysis of Recombinant Bacmid } \\
\hline Primer Pairs & Sequence (5' to 3') & Fragment ${ }^{\text {a }}$ Size (bp) & The Amplified Region \\
\hline pUC/M13 F M2e/ctxB R & $\begin{array}{l}\text { CCCAGTCACGACGTTGTAAAACG CG- } \\
\text { GAATTCTTAATTGCCATACTAATTG }\end{array}$ & 2200 & $\begin{array}{l}\text { Tn7 R + Polyhydrin promoter + M2e/ } \\
\text { ctxB fusion gene }\end{array}$ \\
M2e/ctxB F pUC/M13 R & $\begin{array}{l}\text { CGGGATCCACCATGTCCCTGCTGACCGAGG } \\
\text { AGCGGATAACAATTCACACAGG }\end{array}$ & 1200 & M2e/ctxB fusion gene + Tn7 L \\
pUC/M13 F pUC/M13 R & $\begin{array}{l}\text { CCCAGTCACGACGTTGTAAAACG AGCGGATA- } \\
\text { ACAATTTCACACAGG }\end{array}$ & $\begin{array}{l}\text { Tn7 R + Polyhydrin promoter + M2e/ } \\
\text { ctxB fusion gene + Tn7 L }\end{array}$ \\
\hline M2e/ctxB F M2e/ctxB R & $\begin{array}{l}\text { CGGGATCCACCATGTCCCTGCTGACCGAGG } \\
\text { CGGAATTCTTAATTTGCATACTAATTG }\end{array}$ & 460 & M2e/ctxB fusion gene
\end{tabular}

${ }^{\mathrm{a}}$ All the Fragment Sizes are Expressed Approximately and Has Been Calculated According to the Sequence date of the Bacmid DNA
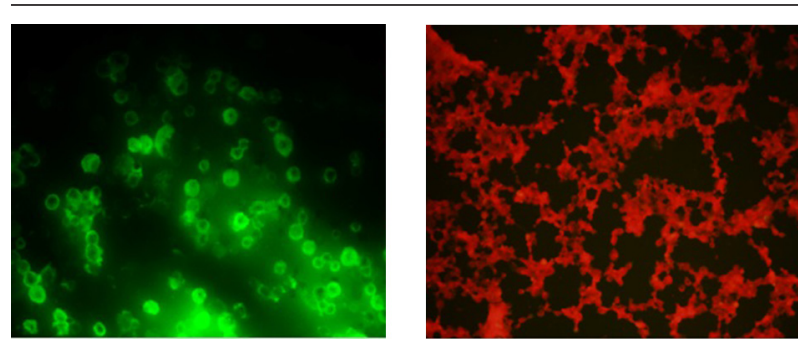

Figure 3. Verification of Recombinant Bacmid Construction Containing M2e-ctxB Fusion Gene Using Indirect Immunofluorescence Sf9 Cells Expressing M2e-ctxB (A), Sf9 Cells Containing non-Recombinant Bacmid DNA as Negative Control (B)

\section{Discussion}

Most influenza vaccines are based on influenza surface glycoproteins hemagglutinin (HA) and neuraminidase (NA). These antigens are subjected to antigenic drift and antigenic shift resulting in epidemic and pandemic influenza around the world. The most recent pandemic occurred in 2009 due to H1N1 influenza A virus. All continents were affected by this new strain, which had enormous economic consequences. The mortality rate varied in different countries; for example in Iran, of the 3672 confirmed cases of influenza, 140 deaths were reported $(21,22)$. Genetic stability is the most important reason for increasing interest on matrix 2 ectodomain domain (M2e) in developing new flu vaccines $(23,24)$. M2e cannot potentially stimulate human immune system; however, immunological responses may increase when we use it along with a potent immunogen. In most of these cases, $\mathrm{M} 2 \mathrm{e}$ is genetically fused to some other proteins. The proteins could be a single antigen such as rotavirus NSP4 (25) or a composition of different particles. Hashemi et al. fused M2e gene to the gene encoded capsid protein of T7 bacteriophage and expressed them in E. coli. M2e-T7 fusion particle enhanced the immunogenicity of mice against influenza virus successfully (26). Cholera toxin subunit B (CtxB) in the form of fusion gene can effectively enhance the immune responses against the fused gene $(27,28)$. The sequencing results showed that M2e was successfully attached to ctxB and the M2e-ctxB fusion frag- ment was constructed as a single open reading frame. Razavi et al. constructed HSP70-E7 fusion gene as a tool in $\mathrm{HBV}$ vaccine research (15). Ebrahimi et al. generated a fusion gene containing M2e and HSP70 and cloned into pPICMH cloning vector as a same manner we did. They also used a serine and glycine codon to separate the two adjacent genes (29).

Different systems, including prokaryotic and eukaryotic expression methods, have been used for production of recombinant viral proteins. Hence, choosing a proper and appropriate expression system is very important to produce viral efficient vaccines. Kim et al. used the prokaryotic expression system to produce M2e. They expressed M2e in Escherichia coli and used it along with an inactivated $\mathrm{H} 9 \mathrm{~N} 2$ virus vaccine and evaluated the mice immune response against the influenza virus (30). Different eukaryotic expression systems including yeast and mammalian systems have also been frequently used for production of M2e fusion genes $(28,31)$ Baculovirus expression vector system (BEVS) is one of the most powerful systems to produce recombinant proteins. Use of BEVSs was increased noticeably after production of the first protein (human Beta interferon) in insect cells infected with baculovirus (32). Using BEVS takes advantages of high level production of biologically active and properly folded functional recombinant protein. However, there are some limitations for using BEVS as an expression system due to difficulty of transferring the recombinant baculovirus into the insect cells (33-35). Incomplete influenza viruses are recently taken into the consideration. These defective viruses usually consist of three or more virus structural proteins such as HA, NA and M, which are selfassembled to construct non-infectious particles called virus like particle (VLPs). VLPs are used in vaccine development and most commonly produced by BEVS (36). Rezaei et al. constructed a recombinant bacmid containing HA gene and expressed in Sf9 cell line. The expressed protein was successfully assembled to generate influenza VLPs (37). Safdar et al. used BEVS to produce M2 for safe production of influenza vaccine. They showed that the insect cell-baculovirus production technology is a modern solution for rapid influenza antigen production, and that this technology is particularly suitable for influen- 
za where annual adjustment of the vaccine is required (38). The same results obtained in a study performed by Slepushkin et al. They induced protective immunity in Balb/c mice against influenza A/Ann Arbor/6/60 virus using baculovirus-expressed M2e (39). Once the transfer plasmid (pFastBac HT) is transformed into E. coli DH10Bac, transposition is mediated by the enzymes encoded by the helper plasmid between the mini-Tn7 element on the pFastBac HT and the mini-attTn7 target site on the bacmid to generate a recombinant bacmid. Bacmid is a shuttle vector replicated in both E. coli and insect cell lines such as SF9 and SF21.

The white and blue colonies appeared on agar plates containing antibiotics, X-gal and IPTG. The appearance of white colonies showed the success of recombinant bacmid construction in DH10Bac cells. Insertion of M2e-ctxB into the mini-attTn7 site of bacmid DNA disrupted the sequence and subsequently prevented expression of LacZ $\alpha$ resulted in appearance of white colonies in the presence of X-gal and IPTG. In contrast, blue colonies on agar plates represent the DH10Bac cells harboring unaltered (nonrecombinant) bacmid. Bacmid DNA is normally greater than 135000 bp in size (19). Since restriction analysis of a DNA fragment with this size is difficult to perform. Moreover, this method is not efficient to verify successful transposition of the fused gene into the bacmid. Agarose gel analyzing is recommended to confirm the bacmid DNA extraction. However, due to some limiting factors such as high molecular weight, long electrophoresis and presence of different DNA bands on agarose gel, it is not recommended to verify the insertion of the target gene onto the bacmid.

A PCR reaction using a combination of pUC/M13 and gene specific primers is the most convenient technique to analyze the recombinant bacmid. Mini-attT7 site, the insertion site of target gene, on bacmid DNA is flanked by pUC/M13 priming regions verifying the presence of the target gene. Gel electrophoresis analysis showed that the PCR reaction amplified the region containing M2e-ctxB on bacmid DNA using different combinations of the primers. Such results did not obtain for the non-recombinant bacmid DNA. These results demonstrated that the M2e-ctxB fusion fragment was successfully inserted into the bacmid DNA. The same results achieved by Salmani et al. where the different sizes of PCR products showed that influenza virus M1 gene transposed into the bacmid DNA (40). The final verification of recombinant bacmid construction was performed by indirect immunofluorescence assay using mAb. All the infected Sf9 cells emitted green fluorescence indicating the presence of the recombinant protein containing M2e-ctxB. This assay indirectly showed that the recombinant bacmid was successfully constructed. In this study, a recombinant bacmid was generated using two simple steps in which M2e-ctxB was inserted into Mini-att T7 site of the bacmid DNA. Indeed the first phase, production of M2e-ctxB fusion protein, was successfully performed. We suggest using BEVS to produce fusion proteins due to its simple use and working flexibility. Moreover, using BEVS can produce a large amount of recombinant protein compared to the other expression systems, which is highly recommended for evaluation of new vaccines.

\section{Acknowledgements}

We gratefully acknowledge the Iranian National Influenza Center personnel and faculty members for their constructive assistance for performing this study.

\section{References}

1. WHO Position Paper on Influenz a Vaccines: Selected references, 2005. Available from: http://www.who.int/immunization/documents/Influenza_refs_final.pdf.

2. Bright RA, Shay DK, Shu B, Cox NJ, Klimov AI. Adamantane resistance among influenza A viruses isolated early during the 2005-2006 influenza season in the United States. JAMA 2006;295(8):891-4.

3. Hehme N, Colegate T, Palache B, Hessel L. Influenza vaccine supply: building long-term sustainability. Vaccine. 2008;26 Suppl 4:D23-6.

4. Global pandemic influenza action plan to increase vaccine supply, Vaccines and Biologicals Epidemic and Pandemic Alert and Response. 2006. Available from: http://whqlibdoc.who.int/hq/2006/who_ ivb_06.13_eng.pdf.

5. Cunha BA. Influenza: historical aspects of epidemics and pandemics. Infect Dis Clin North Am. 2004;18(1):141-55.

6. Kuiken T, Fouchier R, Rimmelzwaan G, Osterhaus A. Emerging viral infections in a rapidly changing world. Curr Opin Biotechnol. 2003;14(6):641-6.

7. Nicholson KG, Webster RG, Hay AJ. Genetic Reassortment of Human Influenza Viruses in Nature, Textbook of Influenza. 1 st edOxoford, UK: Blackwell Science; 1998

8. International Federation of Pharmaceutical Manufacturers \& Associations. Influenza vaccine supply international task force. Technical briefing for WHO intergovernmental meeting on sharing of influenza viruses and access to vaccines and other benefits. 2007. Available from: http://www.ifpma.org/resources/influenza-vaccines/ ifpma-influenza-task-force/about-the-influenza-task-force.html.

9. Galarza JM, Latham T, Cupo A. Virus-like particle (VLP) vaccine conferred complete protection against a lethal influenza virus challenge. Viral Immunol. 2005;18(1):244-51.

10. Palache B. New vaccine approaches for seasonal and pandemic influenza. Vaccine. 2008;26(49):6232-6.

11. Denis J, Acosta-Ramirez E, Zhao Y, Hamelin ME, Koukavica I, Baz $M$, et al. Development of a universal influenza A vaccine based on the M2e peptide fused to the papaya mosaic virus (PapMV) vaccine platform. Vaccine. 2008;26(27-28):3395-403.

12. Holsinger LJ, Nichani D, Pinto LH, Lamb RA. Influenza A virus M2 ion channel protein: a structure-function analysis. J Virol. 1994;68(3):1551-63.

13. Huleatt JW, Nakaar V, Desai P, Huang Y, Hewitt D, Jacobs A, et al Potent immunogenicity and efficacy of a universal influenza vaccine candidate comprising a recombinant fusion protein linking influenza M2e to the TLR5 ligand flagellin. Vaccine. 2008;26(2):201-14.

14. Pinto LH, Lamb RA. The M2 proton channels of influenza A and B viruses.J Biol Chem. 2006;281(14):8997-9000.

15. Razavi NH, Soleimanjahi H, Fotouhi F, Ghaemi A, Fazeli M. Construction of the Chimeric HSP70-E7 Vector and Evaluation of its Protein Production. Iran J Virol. 2009;3(3):29-34.

16. Areas AP, Oliveira ML, Miyaji EN, Leite LC, Aires KA, Dias WO, et al Expression and characterization of cholera toxin B-pneumococcal surface adhesin A fusion protein in Escherichia coli: ability of CTB-PsaA to induce humoral immune response in mice. Biochem Biophys Res Commun. 2004;321(1):192-6. 
17. Müller D, Bayer K, Mattanovich D. Potentials and limitations of prokaryotic and eukaryotic expression systems for recombinant protein production--a comparative view. Microb Cell Fact. 2006;5(Suppl 1):61.

18. van Oers MM. Vaccines for viral and parasitic diseases produced with baculovirus vectors. Adv Virus Res. 2006;68:193-253.

19. Yamshchikov GV, Ritter GD, Vey M, Compans RW. Assembly of SIV virus-like particles containing envelope proteins using a baculovirus expression system. Virology. 1995;214(1):50-8.

20. Airenne KJ, Peltomaa E, Hytonen VP, Laitinen $\mathrm{OH}$, Yla-Herttuala $\mathrm{S}$. Improved generation of recombinant baculovirus genomes in Escherichia coli. Nucleic Acids Res. 2003;31(17).

21. Gouya MM, Nabavi M, Soroush M, Haghdoust AA, Ghalehee S Hemmati P, et al. Mortality from Pandemic Influenza A(H1N1) in Iran. Iran Red Crescent Med J. 2011;13(10):698-701.

22. Lankarani KB, Sabayan B. H1N1 influenza pandemics 2009: from myths to facts. Iran Red Cres Med J. 2010;12(4):354-7.

23. Ebrahimi SM, Aghaiypour K, Nili H. Sequence analysis of M2 gene of avian influenza virus strain (A/Chicken/Iran/101/98 (H9N2)) as an oil vaccine seed. Iran J Biotech. 2008;6(4).

24. Ebrahimi SM, Tebianian M. Influenza A viruses: why focusing on M2e-based universal vaccines. Virus Genes. 2011;42(1):1-8.

25. Andersson AM, Hakansson KO, Jensen BA, Christensen D, Andersen $P$, Thomsen AR, et al. Increased immunogenicity and protective efficacy of influenza M2e fused to a tetramerizing protein. PLoS One. 2012;7(10)

26. Hashemi H, Pouyanfard S, Bandehpour M, Noroozbabaei Z, Kazemi B, Saelens X, et al. Immunization with M2e-displaying T7 bacteriophage nanoparticles protects against influenza A virus challenge. PLoS One. 2012;7(9).

27. Harakuni T, Sugawa H, Komesu A, Tadano M, Arakawa T. Heteropentameric cholera toxin B subunit chimeric molecules genetically fused to a vaccine antigen induce systemic and mucosal immune responses: a potential new strategy to target recombinant vaccine antigens to mucosal immune systems. Infect Immun. 2005;73(9):5654-65.

28. Schotsaert M, De Filette M, Fiers W, Saelens X. Universal M2 ectodomain-based influenza A vaccines: preclinical and clinical developments. Expert Rev Vaccines. 2009;8(4):499-508.
29. Ebrahimi SM, Tebianian M, Mirjalili A, Paykari H, Varshovi HR, Toghyani $\mathrm{H}$, et al. Fusion and sequence analysis of the influenza A (H9N2) virus M2e and C-terminal fragment of Mycobacterium tuberculosis HSP70 (H37Rv). Arch Razi. 2009;64(2):71-6.

30. Kim EH, Lee JH, Pascua PN, Song MS, Baek YH, Kwon HI, et al. Prokaryote-expressed M2e protein improves H9N2 influenza vaccine efficacy and protection against lethal influenza A virus in mice. Virol J. 2013;10:104.

31. Heinen PP, Rijsewijk FA, de Boer-Luijtze EA, Bianchi AT. Vaccination of pigs with a DNA construct expressing an influenza virus M2-nucleoprotein fusion protein exacerbates disease after challenge with influenza A virus. J Gen Virol. 2002;83(Pt 8):1851-9.

32. Smith GE, Summers MD, Fraser MJ. Production of human beta interferon in insect cells infected with a baculovirus expression vector. Mol Cell Biol. 1983;3(12):2156-65.

33. Belzhelarskaia SN. [A baculovirus expression system for insect cells]. Mol Biol (Mosk). 2002;36(3):371-85.

34. Kidd IM, Emery VC. The use of baculoviruses as expression vectors. Applied Biochem Biotech. 1993;42(2-3):137-59.

35. Li SF, Wang HL, Hu ZH, Deng F. Genetic modification of baculovirus expression vectors. Virol Sin. 2012;27(2):71-82.

36. Margine I, Martinez-Gil L, Chou YY, Krammer F. Residual baculovirus in insect cell-derived influenza virus-like particle preparations enhances immunogenicity. PLoS One. 2012;7(12).

37. Rezaei F, Mirshafiey A, Shahmahmoodi S, Shoja Z, Ghavami N Mokhtari-Azad T. Influenza Virus-like Particle Containing Two Different Subtypes of Hemagglutinin Confers Protection in Mice Against Lethal Challenge With A/PR8 (H1N1) and A/HK (H3N2) Viruses. Iran Red Crescent Med J. 2013;15(1):75-82.

38. Safdar A, Cox MM. Baculovirus-expressed influenza vaccine. A novel technology for safe and expeditious vaccine production for human use. Expert Opin Investig Drugs. 2007;16(7):927-34.

39. Slepushkin VA, Katz JM, Black RA, Gamble WC, Rota PA, Cox NJ. Protection of mice against influenza A virus challenge by vaccination with baculovirus-expressed M2 protein. Vaccine. 1995;13(15):1399-402.

40. Salmani Z, Behzadian F, Fotouhi Chahooki F, Fallah J. Construction of a Recombinant Bacmid DNA to Express Influenza Virus Matrix Protein1 (M1) in Insect Cell Line. Iran J Virol. 2010;4(3):23-8. 